\title{
Philippinen: Die Anatomie des Aufstiegs und Niedergangs einer Diktatur
}

\author{
Von Rainer Werning
}

\section{Vorbemerkung}

Im Februar 1986 stürzte mit Ferdinand Edralin Marcos einer der am längsten amtierenden Staatschefs in Südostasien. In den beiden Dekaden seiner Herrschaft verfolgte das Regime in Manila einen politischen und wirtschaftlichen Kurs, dem es das Potential zuerkannte, den Archipel aus Unterentwicklung und Rückständigkeit herauszuführen. In diesem Sinne wurden analog der bereits in Singapur, Hongkong, Taiwan und Südkorea praktizierten exportorientierten Entwicklungsstrategie spin off-Effekte antizipiert, die über allgemeine Einkommenssteigerungen und gerechtere Landverteilung hinaus sicherheitspolitisch durchschlagen sollten.

Solange Manila imstande war, diesem Modell eine ausreichende Legitimation zu verschaffen, genoß es seitens ausländischer Investoren uneingeschränkte Unterstützung. Interne Machtverhältnisse sowie die Dominanz spezifischer Produktionsweisen waren wesentlich verantwortlich dafür, daß spätestens seit der Ermordung des einstigen Ex-Senators Benigno Aquino (21. August 1983) ein Prozeß gesellschaftlicher Polarisierung einsetzte, der mit dem Sturz des Diktators, wenngleich nicht beendet, so doch einen vorläufigen Höhepunkt markierte.

Die Hypothek diktatorialer Strukturen und ökonomischer Erschütterungen lastet schwer auf einer Regierung, die sich der nationalen Aus- und Versöhnung verschrieben hat. Ob und in welchem Maße dies der neuen Präsidentin, Frau Corazon Aquino, gelingt, hängt von der Fähigkeit ab, Rahmenbedingungen für eine genuine Partizipation der Bevölkerung an politischen, wirtschaftlichen wie kulturellen Entscheidungsprozessen zu schaffen, deren Negation allzu lange ausdrücklich als Schlüssel erfolgverheißender Entwicklung galt.

Die vorliegende Untersuchung analysiert die für das Aufkommen und den Fall der Marcos-Diktatur konstitutiven Faktoren und spürt der Frage nach, inwieweit die Februarereignisse eine Zäsur hinsichtlich eines demokratischen Neubeginns setzten.

Nebst Thailand und den Philippinen zählen Malaysia, Singapur, Indonesien und (seit 1984) das Sultanat Brunei zu den Mitgliedstaaten der 1967 in Bangkok geschaffenen Vereinigung südostasiatischer Nationen, ASEAN. 


\section{Beginn der Ära Marcos}

Seit der Unabhängigkeit des Landes (Sommer 1946) bildeten die Liberal Party und die Nacionalista Party die Hauptkräfte im politischen Spektrum des Landes, deren Spitzenkandidaten alternierend in den Malacañang-Palast zu Manila einzogen. Ferdinand E. Marcos, von Haus aus Jurist, war langjähriges Mitglied der Liberalen, bis er, für damalige Verhältnisse wenig aufsehenerregend, ins Lager der oppositionellen Nationalisten überwechselte und als deren Präsidentschaftskandidat 1965 gegen seinen plötzlichen "Widersacher" Diosdado Macapagal das Rennen machte.

Seine Antrittsrede im Januar 1966 war von einer euphorischen Aufbruchsstimmung geprägt. Die Inseln, so Marcos, schickten sich nunmehr an, "eine große Nation" zu werden. Die präsidialen Zielsetzungen folgten einem dem "Zeitgeist" entsprechenden Entwicklungsmodell. Zwecks Eingliederung des Landes in den Weltmarkt wurde auf das Pferd der Exportorientierung gesetzt. Dadurch vermöchte ein rückständiges Land wie die Philippinen sich der zum Anschluß an das internationale Entwicklungsniveau benötigten Technologien zu bemächtigen und in Zeitraffermanier jene Entwicklung nachzuholen, der die westlichen Industrienationen ihren Fortschritt verdankten. Die Vorteile dieser Strategie würden sich in einer Verbesserung der allgemeinen Lebensbedingungen sowie in einem für alle durchsickernden Einkommenswachstum kristallisieren.

So bestechend dieses Modell auf den ersten Blick aussah, so notwendig war es, diesen übrigens nahtlos an den Liberalisierungskurs Macapagals anknüpfenden ökonomischen Richtlinien ein politisches Fundament zu verschaffen. Gerade darin ließen sich die wesentlichen Strukturunterschiede der Marcos-Ära vis-à-vis sämtlichen Vorläuferregierungen dingfest machen. Um dem seit 1966 Gültigkeit beanspruchenden Modell überhaupt reale Erfolgsaussichten einzuräumen, bedurfte es zumindest einer stark zentralistisch ausgerichteten Steuerungsinstanz: der Staatsapparat - vor allem Armee und Polizei mußten gestrafft werden, um die jetzt richtungsweisenden Entwicklungsleitlinien praktisch und möglichst stromlinienförmig umzusetzen. ${ }^{1}$

\section{I.1 Zentralisierung der Staatsmacht}

In diesem Sinne stärkte die Administration die Rolle des Militärs, befürwortete die engere Verbindung zwischen den Polizei- und Konstablereinheiten und schuf ihr unterstellte zentrale Wirtschaftsplanungs-Behörden, die in erster Linie mit den an amerikanischen Eliteuniversitäten geschulten Finanz- und Wirtschaftsexperten bestückt wurden.

Der Vorteil, diese kosmopolitisch orientierten, sich als dynamische Technokraten begreifende Elite an die Exekutive zu binden, bestand zweifellos darin, daß sie selbst sich als überparteilich und unpolitisch begriff. Sie verfügte über keinerlei Massenbasis und

1 Diesbezügliche programmatische Aussagen lief ert u.a. Lucian W. Pye, Armies in the Process of Political Modernization, in: John J. Johnson (ed.), The Role of the Military in Underdeveloped Countries, Princeton 1962, S. 69-89. 
hegte keine eigenen politischen Ambitionen, ja wertete ganz im Sinne ihrer akademischen Leitbilder aus den amerikanischen und europäischen politik- und wirtschaftswissenschaftlichen Fakultäten (partei)politische Aktivitäten als "unnützen Ballast", als "Politikasterei", die den ehrgeizigen wirtschaftspolitischen Plänen nur Knüppel zwischen die Beine würfe. Der Regierung konnte an einer solchen Unterstützung nur gelegen sein. Gerade im Frühstadium der exportorientierten Entwicklungsstrategie war die Expertise dieser "neuen" Elite nicht nur gefragt; die in den Anrainerstaaten verfolgten Modelle schienen ihr recht zu geben und der theoretischen Begründung gegen mögliche Einwände den Glanz des Erfolgs zu verleihen. ${ }^{2}$

Zudem war nicht zu vergessen, daß diese Entwicklungsstrategie ein ideologisches Vakuum ausfüllte, das angesichts ernüchternder Alltagserfahrungen und wachsender Schwierigkeiten in zahlreichen unabhängig gewordenen Ländern aufgerissen worden war. Schlagworte wie Nationalismus, Eigenständig- und Unabhängigkeit, Blockfreiheit etc. mußten, um längerfristig Gültigkeit beanspruchen zu können, mit Inhalt gefüllt werden. Und eine Wirtschaftspolitik, die allen alles versprach, wachstumsorientierte Entwicklung als erstrebenswertes Ziel an sich propagierte, übernahm eine nicht zu unterschätzende ideologische Funktion. Kein Wunder, daß der - seinerzeit keine negativen Konnotationen transportierende - Begriff developmentalism mehr als nur das weite Feld wirtschaftlicher Erneuerungen abdeckte, sondern als "Entwicklung-ismus" Faszination ausstrahlte.

Mit amerikanischer Hilfe wurde in den 60er Jahren das aus Vietnam zurückbeorderte Civic Action-Kontingent (Philcag) der philippinischen Armee mit dem Aufbau von $65 \mathrm{Ci}$ vic Action Centers betraut. Es handelte sich dabei um vom Militär in den ländlichen Gebieten eingepflanzte, auf Bürgernähe bedachte zivile Projekte, die als Zuckerbrot-Variante im übergeordneten Counterinsurgency (A ufruhrbekämpfungs)-Konzept fungierten. Impfaktionen und kostenlose Nahrungsmittelausgaben wurden durchgeführt, um "Herzen und Hirne der Bevölkerung" vorrangig dort zu gewinnen, wo das aus erdrückenden Feudalverhältnissen gespeiste bäuerliche Protestpotential periodisch für "Unruhen" sorgte. Vor allem in Zentralluzon, der Reiskammer und traditionellen Hochburg machtvoller Bauernrevolten, waren nicht weniger als 20 ländliche Gesundheitsteams und 22 Baubataillone der Armee unter den Argusaugen von US-Spezialeinheiten (Green Berets) im Einsatz. ${ }^{3}$

2 Das Journal of the American Chamber of Commerce in the Philippines (JACCP) hatte mehrfach die Technokraten in der Regierung gelobt. Vor allem in seinen Leitartikeln in den Jahren 1970/71 äußerte es sich zuf riedenstellend über Finanzminister (und seit Sommer 1981 außerdem zum Premierminister avancierten) Cesar Virata und Zentralbankgouverneur Gregorio Licaros. Beide hatten maßgeblichen Anteil an der Durchsetzung der dem Land 1970 vom Internationalen Währungsfonds (IWF) verordneten Maßnahmen - Pesoabwertung, beschleunigte Auslandsinvestitionen und Einfrieren der Löhne. Im Juni 1971 konstatierte das JACCP: "Sie (die Technokraten; R.W.) teilen mit der amerikanischen Finanz- und Geschäftswelt die mehr oder minder gleichen Ansichten. Noch kürzlich erklärte der Vorsitzende des Nationalen Wirtschaftsrates: 'Wir sprechen dieselbe Sprache und verstehen uns gut.، " (S. 3).

3 Ausführlich habe ich das an anderer Stelle diskutiert: Rainer Werning, Zur Geschichte des philippinischen Befreiungskampfes: 1930-1975, Bonn 1975 und ders., Philippinen: Aspekte der ländlichen Entwicklung und Weltmarktintegration seit 1972, Münster 1984. 
Allein im Rahmen des 1951 geschlossenen amerikanisch-philippinischen Sicherheitsund Beistandspakts war eine diesbezüglich eng koordinierte Planung und Durchführung militärischer Aktionen vereinbart. Auf amerikanischer Seite zeichnete dafür die Vereinte US-Militärberatungsgruppe (JUSMAG) mit Sitz in Quezon City verantwortlich. Berühmt wurde diese Gruppe durch die Zerschlagung der Hukbalahap $p^{4}$ in den 50er Jahren, als hochrangige US-Offiziere (wie der CIA-Verbindungsmann, Oberst Edward G. Lansdale) die Planspiele für militärische Operationen entwarfen, denen die vom damaligen Verteidigungsminister und späteren Präsidenten Ramon Magsaysay bef ehligten philippinischen Streitkräfte (AFP) das Rückgrat einzogen. Diese Erfahrungen wurden in Vietnam "durchgespielt", wie denn auch die dort vorgenommenen "Verfeinerungen" neuerlich auf dem Archipel zur Anwendung gelangten. ${ }^{5}$

1969 hatte das US-Senatskomitee für Auswärtige Angelegenheiten unter Vorsitz von Senator Symington einen Ausschuß zur Untersuchung amerikanischer Sicherheitsabkommen und Verpflichtung in Ubersee gebildet. Der so entstandene Symington Report enthielt u. a. bemerkenswerte Passagen über die Verhältnisse in den Philippinen. Zitiert wurden darin Auszüge des Roces Committee des philippinischen Abgeordnetenhauses, wonach JUSMAG-Chef General George Pickett Order gegeben hatte, "die AFP unter die Befehlshoheit der JUSMAG zu stellen, dabei die Tatsache übersehend, daß die AFP nicht Teil der amerikanischen Armee sind." "Mit anderen Worten", so resümierte Senator Symington, "wir zahlen der philippinischen Regierung Gelder, um uns vor dem philippinischen Volk zu schützen, das die Amerikaner nicht mag. ${ }^{6}$

4 Im März 1950 erfolgte die Umbenennung der Hukbalahap (Antijapanische Volksbef reiungsarmee, Ende März 1942 in Zentralluzon gegr.) in Volksbefreiungsarmee, HMB.

5 Berechtigterweise liefern die Philippinen und Vietnam ein Lehrstück "konterrevolutionärer Reziprozität ". Jahrelang haben sich amerikanische Sozialwissenschaftler und Militärstrategen intensiv mit dem Problem befaßt, wie die "Huk-Erfahrungen" für Südvietnam und umgekehrt die dort gesammelten "Vietcong-Erfahrungen" für die Philippinen der 70er/80er Jahre optimal - d. h. im Sinne amerikanischer Außen- bzw. Sicherheitspolitik - umsetzbar sind. Der interessierte Leser sei hier auf die - allerdings schwerzugänglichen streckenweise kafkaesk argumentierenden Quellen verwiesen:

a. Guy J. Pauker, Notes on non-military measures in control of insurgency. Sta. Monica/California: Rand Corporation 1962, 12 1., Rand Corp. Papers, P-2642;

b. A.H. Peterson/G.C. Reinhardt/E.E. Conger (eds.), Symposium on the Role of Airpower in Counterinsurgency and Unconventional Warfare: the Philippine Huk Campaign. Sta. Monica/Ca.: Rand Corp. 1963, 61 1. Rand Corp. Memorandum RM-3652-PR (prepared for the U.S. Air Force);

c. Edward G. Lansdale, Viet Nam: Do We Understand Revolution?, in: Foreign Affairs 43.1(Oct. 1964): 75-86;

d. Edward J. Mitchell, The Huk rebellion in the Philippines: an econometric study. Sta. Monica/Ca.: Rand Corp. 1969, 22 1. Rand Corp. Memorandum RM-5757-ARPA;

e. Harvey Averch/John Koehler, The Huk Rebellion in the Philippines: Quantitative Approaches (prepared for the Advanced Research Projects Agency.) Sta. Monica/Ca.: Rand Corp. (August) 1970, 33 p. Rand Corp. Memo. RM-6254-ARPA;

f. Douglas F. Loveday, The role of U.S. military bases in the Philippine economy. Sta. Monica/Ca.: Rand Corp. 1971, 60 p. Rand Corp. Memo. RM-5801-ISA sowie

g. H. Averch/J. Koehler, Explaining Dissident Success: The Huks in Central Luzon. N.p., Jan. 1972, 38 p. Kritisch setzen sich damit u. a. Chomsky und Herman auseinander - vgl. Noam Chomsky/Edward S. Herman, The Political Economy of Human Rights (2 Vols.). Boston 1979.

6 Hearing am 1. Oktober 1969, Symington Report. Washington, D.C.: U.S. Government Printing Office, S. $244 / 5$. 
1947 hatten die philippinischen Militärausgaben knapp 80 Mio. Pesos betragen. 1952 machten sie 142 Mio. aus und kletterten im Jahre 1959, nachdem den Huks erklärtermaßen das Rückgrat gebrochen worden war, auf annähernd 192 Mio. Pesos. Im ersten Haushaltsjahr der Marcos-Administration (1966/67) erreichten die Militärausgaben bereits beachtliche 366 Mio. Pesos und schossen im Haushaltsjahr 1969/70 auf 670 Mio.

Besuchs- und Ausbildungsprogramme für philippinische Offiziere in renommierten USMilitärakademien wie West Point und Fort Bragg wurden arrangiert. Um das Zusammenspiel der Teilstreitkräfte untereinander effizienter zu gestalten, wurden die AFP mit modernem Kommunikations- und nachrichtendienstlichem Gerät ausgestattet. Gleichzeitig bemühte sich die Marcos-Regierung, den ausgeprägten regionalistischen Unterschieden einen Riegel vorzuschieben, indem die Kommandohöhen mit Offizieren aus verschiedenen Landesteilen besetzt und somit eine delikate Balance hergestellt wurde, die sich später zur Herrschaftssicherung als vorteilshaft erweisen sollte.

Durch all diese Schritte wurde nicht nur eines der entscheidendsten Instrumente des Staatsapparates gestrafft. Die praktischen Erfahrungen im Rahmen der Civic ActionProgramme sowie die durch systematische Trainingskurse vermittelten Managementmethoden und Führungseigenschaften auch und gerade im Bereich infrastruktureller Projektbetreuung qualifizierten das Militär für die Wahrnehmung erweiterter staatstragender Funktionen, die sich für zentralistische wirtschaftspolitische Richtlinien instrumentalisieren ließen.

Flankiert wurden diese neuerworbenen Qualifikationen durch militärische Einsätze gegen Bauern, Arbeiter und Studenten in Stadt und Land, die häufig der legislativen Kontrolle (dem Kongreß) entrückt blieben.

Gleichermaßen gestärkt wurden die Polizeieinheiten. 1966 wurde zwischen Malacañang und der US-Botschaft in Manila ein Programm erarbeitet, wonach die polizeilichen Ausbildungsfunktionen von der Amerikanischen Internationalen Entwicklungsbehörde (USAID) wahrzunehmen waren. ${ }^{7}$ Unter der Kategorie "technische Hilfe" finanzierte die USAID das Büro für Öffentliche Sicherheit (OPS), welches maßgeblich an der "Umgestaltung, Finanzierung und Ausbildung des philippinischen Polizeiapparates auf den Philippinen wie in den USA (. . ) beteiligt war. ${ }^{8}$

Im Dezember 1966 wurde Frank E. Walton, gerade aus Südvietnam zurückgekehrt, USAID/OPS-Teamleiter. In Vietnam hatte Walton "für den Aufbau und die Reorganisierung der südvietnamesischen Polizeikräfte verantwortlich gezeichnet - des umfassen-

7 Die AID, wie die CIA unmittelbar dem Präsidenten unterstellt, fördert die Investitionsbedingungen für US-Kapital in den Entwicklungsländern u. a. durch den Auf- und Ausbau der Verwaltung und Polizei. Sie koordiniert ihre Aktivitäten mit der. Entwicklungshilfe des Pentagon. Zudem übernimmt die Behörde für amerikanisches Kapital die Garantie gegen Verstaatlichungen und Verluste infolge von Kriegseinwirkungen. Investitionen und Kosten, ohne die Kapitalinvestitionen in Entwicklungsländern wenig profitabel sind, werden so aus Steuermitteln finanziert.

8 Geoffrey Arlin, The Organisers, in: Far Eastern Economic Review (Hongkong) vom 2. Juli 1973, S. 16 und Elaine Elinson, U.S. Train Counter-Insurgency Police for the Philippines, in: Pacific News Service, (415-986-5690) San Francisco 1971. - Das OPS war während der Präsidentschaft John F. Kennedys (1962) geschaffen worden. 
den CIA-Plans, die politische Infrastruktur der NLF (Nationalen Befreiungsfront; R.W.) aufzulösen. $"{ }^{9} \mathrm{Ihm}$ zur Seite standen Personen mit ähnlichen Erfahrungen aus Brasilien und Äthiopien. Hinzu kamen philippinische Geheimdienstoffiziere, welche die CIA während der Huk-Bekämpfung trainiert hatte und die "Experten auf dem Gebiet der Gegenspionage in und um Saigon (heute Ho Chi Minh-Stadt; R.W.) geworden waren. ${ }^{10}$

Der im Februar 1967 von der Walton-Gruppe fertiggestellte Bericht führte zur Einbindung des AID/OPS-Programms in das CORDS, ${ }^{11}$ dessen philippinische Variante personell mit "alten Hasen" aus Vietnam bestückt wurde. Mit Thomas Rose und Richard Kriegel traten Männer an dessen Spitze, die zuvor Chef der AID-Administration in Saigon bzw. CORDS-Berater in der Provinz Bin Dinh gewesen waren.

Außerdem war seit der Zeit mit dem Aufbau eines integrierten städtischen "Aufruhrbekämpfungs"-Instruments begonnen worden, das schließlich im Großraum Manila als Hauptstadt-Kommando (Metropolitan Command, Metrocom) konkrete Gestalt annahm. Bis zu Beginn der 70er Jahre sind ferner auf amerikanische Initiative neun regionale Polizeiausbildungs-Akademien errichtet und acht städtische Polizeihauptquartiere, mit technischen Hilfen und kompletten Trainingsprogrammen versehen, geschaffen worden. Bis zum Sommer 1971 waren auf diese Weise 10540 Polizisten, 60 Techniker und knapp 1900 Radiofachleute ausgebildet worden. ${ }^{12}$

Für die Durchsetzbarkeit der exportorientierten Entwicklungsstrategie war die Unterstützung bedeutsamer Teile der Geschäftswelt vonnöten. Der traditionelle Exportsektor, jene Unternehmen, die von der "Nationalisierung" bestimmter Geschäftszweige auf Kosten der Chinesen (z. B. Handel) profitierten, sowie die mit ausländischem Kapital liierten Firmen versprachen sich von der neuen Politik eine institutionell abgesicherte Wahrung ihrer Interessen. Teile der nationalen Bourgeoisie bezogen eine abwartende Position, während andere, der sich nationalistisch gebenden Regierungspropaganda Glauben schenkend, ins Marcos-Lager überschwenkten, vor allem dessen rasche "Handhabung" von Arbeitskonflikten schätzten.

Politisch drückten diese Entwicklungen die schleichende Entfremdung von den bestehenden Parteien aus, gegen die der - nicht einmal unberechtigte - Vorwurf adressiert war, eine engstirnige Politik zu betreiben, sich in endlosen Fraktionsquerelen abzuschleifen und persönliche Domänen abzustecken. Demgegenüber galten die mit Marcos verbundenen Technokraten als no nonsense-Beamte, als kompetent und parteipolitisch nicht ambitioniert. Sie fanden in Marcos und dieser in ihnen die kongeniale Entsprechung.

9 Ebda.

10 Ebda. und N. Chomsky/E.S. Herman, The Washington Connection and Third World Fascism - The Political Economy of Human Rights, Vol. 1. Boston 1979.

11 CORDS (Civil Operations and Rural Development Support Program) war solchen "Bef riedungs"programmen wie Phoenix zugeordnet. Es beinhaltete u. a. "selektiven Gegenterror" (CT), um seit Mitte der 60er Jahre die "Ausmerzung" von NLF-Kadern zu betreiben. S. u. a.: Wayne Cooper, Operation Phoenix: A Vietnam Fiasco Seen From Within, in: Washington Post vom 8. Juni 1972 und Indochina Resource Center, A Statistical Fact Sheet on the Indochina War, Berkeley (27. 9. 7.2).

12 U.S. Senate, Committee on Foreign Relations, 93rd Congress, 1st Session, Korea and the Philippines: November 1972. A Staff Report. Washington, D.C. (18. 2.) 1973, S. $38 / 9$ und E. Elinson, op. cit. 
Gleichzeitig entstanden mehrere Management- und Verwaltungsfachschulen - so das College of Business Administration, das College of Public Administration an der University of the Philippines und das Asian Institute of Management. Die Lehrpläne hatten amerikanische Professoren entworfen und wurden z. T. von ihnen selbst abgewickelt. Hatten diese Schulen die für die Durchsetzung der neuen Politik erforderlichen Fachkräfte auszubilden, so leitete die Regierung Ende der 60er Jahre einen darüber hinausgehenden Schritt ein. Der vom Präsidenten eingesetzten Kommission zur Untersuchung des Ausbildungswesens oblag die "umfassende Bestandsaufnahme des (. . .) Erziehungssystems, um festzustellen, ob und inwieweit es im Sinne der nationalen Entwicklungsziele relevant war." ${ }^{13}$

Es ging darum, eine Umorientierung im Ausbildungssektor mit Blick auf die anvisierten infrastrukturellen Großprojekte (z. B. Auf- und Ausbau von Straßen, Brücken, Häfen und Kommunikationssystemen) vorzubereiten. Dies mußte Papier bleiben, solange nicht ein zur Umsetzung dieser Projekte herangebildeter Kader bereitstand.

Die o. g. Untersuchungskommission folgte einem ökonomischen wie politisch-ideologischen Kalkül. Geänderte Curriculum-Pläne ebneten einer "Umsattelung" der Intelligenz den Weg, was seinerzeit hitzige Campus-Debatten entfachte, in denen das Gros der Studenten aus seinen antiimperialistischen Sentiments keinen Hehl machte. In ihren Augen bestand zwischen der ideologischen Manipulation und des auf den Schlachtfeldern Indochinas verübten Genozids ein struktureller Zusammenhang. Neben den Bauern und städtischen (Transport)Arbeitern bildeten denn auch die Studenten die schärfsten Gegner und Kritiker der Marcos-Regierung. ${ }^{14}$

Um den neuen Kurs und die sie begleitende Ideologie wirksam in Schlüsselpositionen staatlicher Macht zu verankern, erhielten die Technokraten Zug um Zug erweiterte Kompetenzen, die vormals von Politikern wahrgenommen wurden. Marcos' Wirtschaftskredo - verstärkte Abhängigkeit von Auslandsinvestitionen; Ermunterung des Freihandels; Beseitigung von Restriktionen für die internationale Geschäftswelt; Aufnahme umfangreicher Auslandsdarlehen; ungehinderte Profittransfers; Reallohnsenkungen etc. - wurde schrittweise umgesetzt und durch die einflußreichsten Unternehmensvereinigungen gutgeheißen. ${ }^{15}$

All diese militärischen, wirtschaftlichen, politischen, administrativen und ideologischen Faktoren zusammengenommen markierten eine Rationalisierung und Zentralisierung staatlicher Herrschaft, die für die Rahmenbedingungen der neuen Politik wie für die Sicherung der materiellen Basis der herrschenden Klassen konstitutiv waren. Natürlich ge-

13 Letizia R. Constantino, World Bank Textbooks: Scenario or Deception. Quezon City(Philippines): Foundation for Nationalist Studies 1983, S. 23.

14 Als First Quarter Storm - so benannt nach der Protest- und Demonstrationswelle seit dem ersten Quartal 1970 - ging diese national orientierte und sich antiimperialistisch begreifende Bewegung in die Annalen ein.

$15 \mathrm{Zu}$ diesen Vereinigungen zählten beispielsweise: Philippine Chamber of Commerce and Industry; Chamber of Agriculture and National Resources; Philippine Chamber of Industries; Private Development Corporation of the Philippines; Presidential Economic Staff; Board of Investments und das American Chamber of Commerce in the Philippines. Nach Verhängung des Kriegsrechts (Herbst 1972) gesellten sich dazu noch die National Economic and Development Authority (NEDA) und Development Academy of the Philippines. 
schah dies nicht über Nacht. Ihr Aufbau vollzog sich schrittweise und systematisch und verlieh der Regierung Glaubwürdigkeit und Anziehungskraft. Wie keinem Präsidenten vor ihm glückte Marcos immerhin im Jahre 1969 die Wiederwahl. In Verbindung mit der von Washington aufgrund der Indochina-Ereignisse anvisierten militärstrategischen Kräfteumgruppierung in der Region begründeten die o .g. Faktoren jene "eisernen" Voraussetzungen für ein Entwicklungsmodell, das sich schließlich 1972 relativ reibungslos des Kriegsrechts zur "prophylaktischen Immunisierung" von Protest und Widerstand bedienen konnte.

\section{I.2 Sozialökonomische Implikationen}

Hatten Marcos' Vorgänger die vormals bestandenen Devisen- und Importbeschränkungen gelockert, so ging seine Regierung einen Schritt weiter. 1967 wurde im Kongreß das Investitionsförderungs-Gesetz verabschiedet, nach dem der Anteil ausländischen Kapitals an lokalen Unternehmen $40 \%$ nicht überschreiten sollte. Unklar blieb, wie dies bei den vornehmlich US-beherrschten Erdölraffinerien, Bergwerksgesellschaften, Außenhandelsfirmen etc. zu realisieren war. Trotz geschickter nationalistischer Rhetorik ${ }^{16}$ vermochten Marcos und seine Technokraten die Aufnahme einer Sonderklausel in dieses Gesetz einzubauen, wonach ausländische Investoren auch dann noch $100 \%$ iger Kapitaleigner sein konnten, wenn sie bekundeten, innerhalb eines Jahrzehnts nach Registrierung der betreffenden Firmen Aktien an Filipinos zu veräußern.

Das kurz darauf ratifizierte Exportförderungs-Gesetz befürwortete gar einen 55\%igen Kapitalanteil ausländischer Firmen in der Export- und einen 100\%igen Anteil in sog. Pionierindustrien. Auf der Halbinsel Bataan entstand der Inseln erste Freihandels- und Exportproduktionszone Mariveles, in der philippinische Steuergesetze nur bedingt griffen. ${ }^{17}$

US-Konzerne und ihre philippinischen Töchter vereinigten mehr als die Hälfte aller Geschäftsanlagen auf sich, deren Buchwert Ende 1969 nach Schätzungen des philippinischen Nationalen Wirtschaftsrates (NEC) 2 Mrd. US-Dollar betrug. Im Vergleich zu der vom NEC 1965 durchgeführten Buchwertaufstellung von US-Anlagen, die mit mindestens 1,15 Mrd. US-Dollar ausgewiesen worden und von denen allein 807 Mio. Dollar

16 In diesem Sinne operierte Marcos periodisch mit der - letztlich leeren - Drohung, sich der Sowjetunion anzunähern, sollten bestimmte an Washington adressierte "Forderungen" unerfüllt bleiben. Bei den Basenverhandlungen $(1979,1983)$ beispielsweise pokerte er mit solchen Argumenten um umfangreichere Militärund Wirtschaftshilfe, während seine Tochter Imee in ihrer Eigenschaft als Vorsitzende der Kabataang Barangay (sie verstand sich als Jugendorganisation der Marcos-Partei Kilusang Bagong Lipunan = Bewegung der Neuen Gesellschaft) gegen den "US-Imperialismus" wettern durfte.

17 Anfang der 70er Jahre entstand dort die erste Exportproduktionszone im Lande - die Bataan Export Processing Zone (BEPZ) -, der bald weitere in Baguio City, auf Mactan und in Davao City folgten. Noch bis Ende der 70er Jahre wurde von Planungsstäben des Präsidenten anvisiert, deren Zahl auf 17 zu steigern, was infolge der einsetzenden politischen und wirtschaftlichen Krise und modifizierter Pläne der IWF-WB-Gruppe über das Stadium eines Reißbrettentwurfs nicht hinauskam. 
auf 108 amerikanische Großunternehmen konzentriert waren, lagen die für die nationale Industrieentwicklung negativen Folgen des Investitions- und Exportförderungs-Gesetzes auf der Hand.

Offiziell machten diese Anlagen $60 \%$ aller US-Privatinvestitionen in Südostasien aus, wobei ihr Anteil am Volumen ausländischer Privatinvestitionen auf den Inseln $80 \%$ betrug. Statistiken der Philippinischen Zentralbank wiesen aus, daß von 1960-69 160 Mio. US-Dollar an ausländischen Neuinvestitionen ins Land flossen, gleichzeitig aber 482 Mio. US-Dollar an Profiten außer Landes transferiert wurden. ${ }^{18}$

Vormals für den Inlandbedarf produzierte Verbrauchsgüter verknappten sich und mußten - abwertungsbedingt - durch verteuerte Importe ausgeglichen werden. Eine expansive Geldpolitik der Zentralbank und die Überbetonung infrastruktureller Großprojekte taten ein übriges, um über die Inflation die Kosten der Exportorientierung auch auf philippinische Klein- und Mittelbetriebe abzuwälzen. ${ }^{19} \mathrm{Um}$ aber die auf Export abgestellte Wirtschaft wettbewerbsfähig zu halten, war Manila darauf angewiesen, den Außenhandelswert des Pesos zur Ankurbelung des Handels niedrig zu halten.

Die seinerzeit von der Regierung selbst veröffentlichten wirtschaftlichen Eckdaten veranschaulichten diesen Trend.

Gegenüber 1961-65 erhöhte sich das Haushaltsdefizit in den folgenden fünf Jahren um $72 \%$. Dies war wesentlich Ausfluß ehrgeiziger Infrastrukturprogramme, für die in der zweiten Hälfte der 60er Jahre im Vergleich zur ersten um $92 \%$ gestiegene Finanzmittel alloziert wurden. Geöffnet hatte sich auch die Schere zwischen Investitionen und Spareinlagen: Nach einem jährlichen Uberschuß bei Spareinlagen (1961-65) stellte sich ein Investitionsüberhang von durchschnittlich 294,2 Mio. Pesos (1966-70) ein. Diese Veränderungen widerspiegelten sich in jährlich drastisch gestiegenen Haushaltsdefiziten - von 22 Mio. (1961-65) auf 213,6 Mio. US-Dollar (1966-70). Das resultierte innerhalb der letztgenannten Zeitspanne in einer kurzfristigen öffentlichen Verschuldung um $100 \%$ und eine 84 \%ige Zunahme der Auslandsverschuldung von 447 Mio. auf 822 Mio. USDollar. ${ }^{20}$

Dieses Muster blieb in der Folgezeit bestimmend: Die "öffentliche Hand" trieb die kurzfristigen Schulden zwischen 1966-70 und 1971-75 nochmalig um das Doppelte in die Höhe, während die Auslandsschuldenlast im Vergleichszeitraum gar von 822 Mio. auf 2,586 Mrd. US-Dollar wuchs. Finanzlöcher wurden zunehmend durch Auslandskredite zugestopft. Diese Darlehen wurden den politisch aufs engste mit der Regierung verflochtenen privaten Geschäftsleuten überantwortet (cronyism), die damit sowohl eigene, vornehmlich im Exportbereich angesiedelte Firmen aufbauten oder sich, was weitaus häufi-

18 Das ist vom Autor an anderer Stelle ausführlich diskutiert: Rainer Werning, Verhinderte Unabhängigkeit Agrar- und Wirtschaftspolitik in den Philippinen. Münster 1985, S. 223-8.

19 Ebda.

20 Angaben der NEDA, Philippine National Account Statistics und Key Indicators of Developing Member Countries of the A(sian) D(evelopment) B(ank) vom April 1984. - Zit. nach: Emmanuel S. De Dios (ed.), An Analysis of the Philippine Economic Crisis, A Workshop Report. Quezon City: University of the Philippines, Juni 1984 , S. $11 / 2$. 
ger geschah, mit dem Auslandskapital liierten. Die Unterscheidungslinien zwischen Staat, Regierung, bestimmten lokalen (d. h. Marcos politisch nahestehenden) Großgrundbesitzern wie Geschäftsleuten und ausländischem Kapital wurden zunehmend verwischt.

Gewiß führen im Vergleich zum Bruttosozialprodukt hohe Haushaltsdefizite, wie das japanische Beispiel eindrücklich belegte, nicht automatisch zu galoppierender Auslandsverschuldung. ${ }^{21}$ Haushaltsdefizite sind ohne den Rückgriff auf erhöhte Darlehen aus dem Ausland nur mittels einer durch Produktivitätssteigerungen abgesicherten Ausgabenpolitik zu stoppen, wobei die Ressourcenverwendung in den produktivsten Wirtschaftssektoren Voraussetzung ist.

Das aber war nicht der Fall. Während in den meisten Anrainern die Arbeitsproduktivität in den 60er und 70er Jahren Zuwächse von jährlich durchschnittlich $6 \%$ erreichten, waren es in den Philippinen weniger als $2 \%$ und hinkten damit nicht nur in Ostasien, sondern auch innerhalb der ASEAN, ja selbst im Vergleich zu Indien und Nepal hinterher. ${ }^{22}$ Schließlich verwies das Problem mangelnder Produktivitätssteigerung in dem agrarisch ausgerichteten Land auf die besonderen Klassen- und Besitzkonstellationen, die - vor allem im Nahrungsmittelbereich - feudalistisch geprägt waren. ${ }^{23}$

1970 veröffentlichte die Wirtschaftsplanungsbehörde des Kongresses (CEPO) Daten, welche die Einkommensverteilung als extrem unausgeglichen auswiesen. Danach bezogen $194810 \%$ der Familien 30 \%, 1961 aber schon $46 \%$ des Nationaleinkommens. 1965 besaßen $5 \%$ die Spitzenverdiener soviel wie die am untersten Ende der Skala angesiedelten $60 \%$. Im Jahre 1956 hatte das untere Fünftel der Familien 4,5\% der Einkommen auf sich vereinigt, während ihr Anteil 1965 auf 3,5\% schrumpfte.

Berücksichtigte man die Realeinkommen, so war die Kluft noch offenkundiger: 1969 hatten über $50 \%$ aller Familien Jahreseinkommen von weniger als 1500 Pesos. Die Durchschnittsfamilie mit sechs Mitgliedern verfügte damit über ein durchschnittliches jährliches Pro-Kopf-Einkommen von 250 Pesos. Nach Angaben der New York Times aus dem Jahre 1970 betrug das mittlere Einkommen im Land umgerechnet 50 US-Dollar pro Jahr. Die Schwierigkeiten bei der Schätzung des tatsächlichen Ausmaßes der Einkommensverteilung lagen darin begründet, daß es in der unteren Einkommensklasse ca. eine Million Familien gab, die keine bzw. kaum Bareinkommen bezogen.

In den ländlichen Gebieten, in denen damals etwa $75 \%$ der Filipinos lebten und in denen $60 \%$ aller Erwerbstätigen beschäftigt waren, lagen die Einkommen erheblich unter dem Durchschnitt. 1968 betrug das durchschnittliche Jahreseinkommen einer Landfamilie 400 Pesos. Der Anteil der Farmen unter Pacht, der vor $194635 \%$ und $194837 \%$ be-

21 Vgl. u. a. T. C. Smith, Agrarian Origins of Modern Japan. Stanford University Press 1959.

22 Harry T. Oshima, Sector Sources of Philippine Postwar Growth, in: Journal of Philippine Development (Manila), 1st Semester 1983, S. 9, Tab. 2.

23 Vgl. dazu: Rainer Werning, Agrarreform und Kriegsrecht auf den Philippinen -Anatomie einer Pazifizierung. Münster 1983 und Rolf Hanisch, Der Staat, ländliche Armutsgruppen und legale Bauernbewegung in den Philippinen. Baden-Baden 1983. 
tragen hatte, war 1970 auf $52 \%$ hochgeschnellt - trotz Verabschiedung erlassener Landreformgesetze. ${ }^{2}$

Nach den im Jahre 1960 erhobenen Landwirtschafts-Statistiken waren $63 \%$ der Reispflanzer Kleinpächter, welche im Durchschnitt 2,6 ha Land bestellten. 1963 gab es unter der damals 27 Millionen Einwohner zählenden Bevölkerung acht Millionen Kleinpächter. Mitunter mußten sie dem Bodenbesitzer bis zu 75-80 \% der Ernteerträge abführen. Das 1963 verabschiedete Landreformgesetz (ALRC) Macapagals sollte das Los der Bauern durch eine Reduzierung der Landpacht auf $25 \%$ verbessern. Die Landbehörde gelangte jedoch später zu der Feststellung, daß bis 1969 lediglich 13377 Pächter aus dem Millionenheer tatsächlich eine nur 25 \%ige Landpacht entrichteten. Das Landreformgesetz hatte sich aufgrund vielfältigster Machinationen der dominierenden Großgrundbesitzerklasse als Flop erwiesen.

Die Landfrage sollte hernach ein innenpolitisch bestimmendes Moment bleiben, deren Lösung als "Eckpfeiler" der mit dem Kriegsrecht verkündeten "Neuen Gesellschaft" bestimmt wurde. ${ }^{25}$ Zumindest war auch und gerade damit seitens der Regierung die Dringlichkeit angesprochen, dem Kriegsrecht einen legitimatorischen Anstrich zu verleihen.

\section{Die Kriegsrechtsperiode (1972-1981)}

Die Proklamation Nr. 1081, mit der Marcos am 21. September 1972 das Kriegsrecht verhängte, markierte den aus Manilas Sicht erfolgreich eingeleiteten Prozeß der Zentralisierung und Konzentration staatlicher Herrschaft. Die skizzierten Strukturmerkmale des neuen Entwicklungsmodells (s. S. 32-4) bedurften fortan lediglich der Multiplizierung und Anwendung gegen ein wachsendes Potential seiner Gegner und Kritiker. Die Instrumente zur Ausschaltung oppositioneller Regungen waren ja bereits vor dem 21. September geschaffen worden.

Gekoppelt mit den Maßnahmen Washingtons, seine Bündnispartner in der Region angesichts des sich abzeichnenden Vietnam-Debakels nicht abdriften, ihnen vielmehr - wenn schon nicht mit Bodentruppen, so doch wenigstens mit Militär- und Materialhilfen ( $\mathrm{Ni}$ xon-Doktrin) - Rückendeckung zu geben, wird die Ausrufung des Kriegsrechts zu dieser Zeit verständlich. Der von Marcos vorgeschobene Grund - Zurückschlagen des Staates gegen eine von "rechten Oligarchen" und "linken Verschwörern"/"sezessionistischen Moslems im Süden" orchestrierte "Subversion" - war so wenig überzeugend wie die These, er habe das Kriegsrecht aus persönlichen Beweggründen zur Ausschaltung seiner politischen Rivalen (vorab Benigno Aquinos) genutzt. ${ }^{26}$

24 Ebda.

25 Ferdinand E. Marcos. Notes on the New Society of the Philippines. Manila: Marcos Foundation, Inc. 1973; ders., Notes on the New Society of the Philippines II. Manila: National Media Production Center 1976 und ders., Revolution from the center: How the Philippines is using martial law to build a New Society. Hongkong 1978.

26 Diese Ansicht ist vor allem von der von Salvador H. Laurel (heute Vizepräsident und Außenminister in der Aquino-Regierung) geführten United Nationalist Democratic Organization (UNIDO) vertreten worden. In ihrer Uberbewertung offenbart sich ein verkürztes; personalistisch gewendetes Geschichtsverständnis. 
Opposition hatte sich seit 1970 unter den Bauern, städtischen (Transport) Arbeitern, Studenten und Teilen der Intelligenz Bahn gebrochen, die aufgrund ökonomischer Benachteiligungen und mangelnder Arbeits- und Berufsperspektiven auf die Straße gingen. So aufgewühlt waren die Wellen des Protests, ${ }^{27}$ daß deren Schaumkronen gar jenen Teil des Staatsapparates erfaßten, dem Marcos bis dato wenig Aufmerksamkeit gewidmet, von dem er sich wohl auch eine zumindest neutrale Haltung versprochen hatte - nämlich die Judikative.

Am 17. August 1972 stufte der Oberste Gerichtshof im sog. Quasha-Fall sämtlichen von Amerikanern unter den Bedingungen der Gleichheitsklauseln erworbenen Grund und Boden als illegalen Besitz ein, der mit Auslaufen des diese Klauseln absichernden Laurel-Langley-Abkommens (4. Juli 1974) automatisch in philippinischen Staatsbesitz übergehen sollte. Am 18. August wurden ausländische Firmen durch den sog. LustevecoFall aufgefordert, in bestimmten Wirtschaftszweigen nur Filipinos in Managementpositionen zu beschäftigen.

Eine der allerersten Maßnahmen des Präsidenten nach seinem Coup bestand in der sofortigen Aufhebung dieser beiden spektakulären Urteile. Wenig später konstatierte ein dem US-Senatskomitee für Auswärtige Angelegenheiten vorgelegter Bericht:

"Wir sind der Meinung, daß, wenn überhaupt, nur wenige Amerikaner die Einschränkung persönlicher Rechte und demokratischer Institutionen als negative Beeinflussung amerikanischer Interessen ansahen ... Amerikanische Beamte sind zudem der Ansicht, daß die Stärkung präsidialer Autorität... Präsident Marcos befähigte, eine gewünschte Stabilität herbeizuführen, die in unserem Interesse erfolgte, und daß . . . die Militärbasen und eine uns wohlgesonnene Regierung in den Philippinen wichtiger als die Wahrung demokratischer Institutionen sind. ${ }^{28}$

Am 9. Oktober 1972 gab Marcos der Zeitschrift U.S.News \& World Report ein Interview, in dem er ausländische Kapitalgeber wie folgt hofierte:

"Wir sind an allen Formen von Kapital interessiert, und ich möchte an dieser Stelle zwei Dinge ausdrücklich betonen: wir werden die großzügigsten Anreize geben, und ausländisches Kapital wird geschützt werden . . . Die Amortisation von Investitionen, Kapitalabfluß und Profittransfers werden garantiert."

In diesem Sinne erfolgte eine kontinuierliche Steigerung von US-Militärhilfen, während der Verteidigungsetat - inklusive der für geheimdienstliche Zwecke bereitgestellten Gelder - um das Zehnfache aufgestockt wurde. Die Militärstreitkräfte wuchsen sich bis heute mit 285000 Soldaten $(1972=62000)$ zu einem formidablen Unterdrückungsapparat aus. Dazu gesellen sich die noch immer intakten Kontingente der Integrierten Nationalpolizei/Constabulary (INP/PC), paramilitärische Verbände wie die Integrierten Bürgerwehrtruppen (ICHDF) sowie vom Militär geförderte bzw. tolerierte pseudo-

28 U.S. Senate. Committee on Foreign Relations, 93rd Congress . ., op. cit., S. 45. (Hervorhebungen; R.W.) 
religiöse Banden und/oder Sekten wie Rock Christ, Lost Command. New Jerusalem und Sigma. ${ }^{29}$

Die Militärgerichtsbarkeit hielt Einzug und selbst wirtschaftliche Planungs- und Infrastrukturmaßnahmen wurden zunehmend der Kompetenz des Militärs überantwortet, die Anfang 1980 immerhin über $50 \%$ aller Präsidialbeamten für regionale Entwicklungsvorhaben (PROD) stellten. ${ }^{30}$

Diese massive Militarisierung folgte ausschließlich einem internen "Sicherheits"kalkül; von außen - wie beispielsweise der ASEAN-Partner Thailand* - hatten und haben die Inseln keinen äußeren Feind zu fürchten. Bislang ist ein Bruch in dieser nationalen "Sicherheits"logik nicht auszumachen: Der seit 1982/83 offiziell gültige Oplan Katatagan (Operationsplan Stabilität) zielt gleich der seinerzeit im Vietnam-Krieg praktizierten Operation Phoenix auf die Aushebelung der "kommunistischen Infrastruktur und Logistik ". Zwischen 1972 und 1984, so schätzte das Philippinische Rote Kreuz, wurden 5,7 Millionen Menschen (mehr als ein Zehntel der Bevölkerung) Opfer von Vertreibungen - städtische Arme, Bauern, ethnische Minderheiten und Moslems im Süden. ${ }^{31}$

Für Manila erfolgte dies im Einklang mit der "nationalen Sicherheit". Der nach 1972 propagierte Regime-Slogan Isang Bansa - Isang Diwa ("Eine Nation - ein Geist") reflektierte die philippinische Variante jener Vorstellungen, die Brasiliens Sicherheitsideologe, General Golbery de Couto e Silva, einst idealtypisch formulierte - "Die Nation ist absolut, oder sie ist nichts. Eine Nation kann keinerlei Begrenzung ihrer absoluten Macht dulden."

Qua Definition galten folglich selbst keimhafter Widerspruch und Protest als unzulässige Infragestellung der monolithisch gesetzten Nation, deren "Zurückschlagen" allseits gerechtfertigt war. Zudem wurde ihm ein geopolitisches Element unterlegt: Dieses betonte die Stärkung nationaler Einheit und staatlicher Macht als unabdingbare Voraussetzung für ein Úberleben im Bezugsrahmen einer virulenten - i. d. F. Ost-West- Blockkonfrontation. In letzter Konsequenz wurden das Fortbestehen des Staates und die Garantie nationaler Sicherheit, wie Marcos stets hervorhob, der Entscheidungsbefugnis der westlichen Führungsmacht USA überantwortet.

Das Militär fungierte dabei als Rückgrat. Seine Rolle war in einem im November 1980 publizierten Counterinsurgency-Handbuch wie folgt zusammengefaßt:

"Die grundlegende Philosophie der nationalen Counterinsurgency bildet die untrennbare Verknüpfung von Sicherheit und Entwicklung. Sicherheit erlaubt die Entwicklung weiträumiger Gebiete; Entwicklung ist der Sicherheit förderlich. Sicherheit und

29 Ausführlich in: Aktionsgruppe Philippinen/medico international (Hrsg.), Die gestutzten Flügel der Freiheit Menschenrechte und Militarisierung in den Philippinen. Münster 1985. Es ist dies die deutsche Ausgabe einer von philippinischen Menschen- und Bürgerrechtsorganisationen herausgegebenen Studie.

30 Alexander R. Magno (ed.). Nation in Crisis: A University Inquires Into the Present. Quezon City 1984.

31 Aktionsgruppe Philippinen/medico international (Hrsg.), Die gestutzten Flügel ..., op. cit. 
Entwicklung müssen Hand in Hand gehen, das eine vermag ohne das andere nichts zu erreichen. ${ }^{32}$

\section{Der Aquino-Mord (1983): Von der gesellschaftlichen Polarisierung zur Isolierung des Regimes}

Nach erlittenen Rückschlägen bis Mitte der 70er Jahre war es der Kommunistischen Partei/ Neuen Volksarmee (CPP/NPA) und der ihr übergeordneten, im Frühjahr 1973 entstandenen Nationalen Demokratischen Front (NDF) gelungen, ihre Operationsbasis auf die mittlere Visaya-Inselgruppe und nach Mindanao auszuweiten. ${ }^{33}$ Der von ausländischer Unterstützung abgeschnittenen Guerilla gelangen aufgrund einer geschickten Verknüpfung von bewaffnetem Kampf und politischer Massenarbeit in den Städten Einbrüche bis tief ins bürgerliche Lager hinein.

Außerdem waren die neben dem Staatsapparat einzig intakt gebliebenen Institutionen, die Kirchen, deren unterer Klerus sich gleichsam Mitte der 70er Jahre auf oppositionelle Positionen begeben hatte, und kirchlich unterhaltene soziale Aktionszentren wiederholt zur Zielscheibe des Regimeterrors geworden. ${ }^{34}$

So sehr Protest wie legaler und illegaler Widerstand wuchsen, so wenig war die Militärspitze unter General Fabian C. Ver der "Aufgabe" gewachsen, der Guerilla das Rückgrat zu brechen. Für das Militär bereitgestellte Gelder versickerten in den Taschen der fat bellies. ${ }^{35}$ Das politische System und die Militärs erwiesen sich in dem Augenblick als dysfunktional, wo ihr Profil und die Legitimation staatlicher Herrschaft dringender denn je vonnöten waren.

Mit der kaltblütigen Ermordung des nach dreijährigem Selbstexil in den USA zurückgekehrten Ex-Senators Benigno Aquino auf dem Flughafen von Manila (21. August 1983) spitzte sich diese Situation zu. Aquino war die Galionsfigur des auf einen friedlichen Wandel spekulierenden Bürgertums, der gemeinsam mit dem Erzbischof von Manila, Kardinal Jaime Sin, beabsichtigte, gegenüber dem Präsidenten einen nationalen Aus-

32 Hrsg. vom Command and General Staff College, Fort Bonifacio, Metro Manila, November 1980 - zit. nach: Katarungan (Manila) 3. 8. (Sept./Okt. 84):8, hrsg. wird die Zeitschrift von der Okumenischen Bewegung für Gerechtigkeit und Frieden. (Hervorhebung; R.W.).

33 In der ersten Ausgabe des internen und theoretischen Organs der CPP (Rebolusyon, 1. Juli 1976), die mit Our Urgent Tasks betitelt war, faßte das Zentralkomitee die Entwicklung der Partei nach dem 2. ZK-Plenum (1971) zusammen. Beim damals beschlossenen Aufbau regionaler Parteikomitees und -organisationen wurden der "Linksopportunismus" als Hauptf ehler diagnostiziert. - S. ferner: Ang Bayan (CPP-Zentralorgan), 0.O., Dezember 1983, S. 1-7.

34 R. Werning, Verhinderte Unabhängigkeit ..., op. cit., S. 90 f.

35 So lautete die abfällige Etikettierung der inkompetenten Militärs, die ihre Karriere einzig der Nähe zum Präsidenten verdankten. Seit 1981 wurden die strategischen Schlüsselstellungen des Militärs, die 13 Regionalkommandos, maßgeblich in die Hände dieser "fat bellies" gelegt, in deren Folge die über die Köpfe länger gedienter Offiziere hinweg erfolgte Benennung von General Ver zum Generalstabschef für Mißstimmung sorgte. Ver hatte sich vom Leibwächter und Chauffeur zum Chef der Malacanang-Palastwache des Präsidenten und schließlich des Geheimdienstnetzes emporgedient. 
und Versöhnungskurs ins Spiel zu bringen. Seine Liquidierung brachte die Protestspirale in Bewegung und dem Bürgertum schlagartig die Menschenrechtsfrage "näher". Hatte dieses mehrheitlich zu dem alltäglichen Staatsterror gegen die Bauern und ethnischen Minderheiten geschwiegen, so propagierte es jetzt die Beseitigung der Marcos-Diktatur und machte sich partiell selbst die weitergehende Losung vom "Sturz der US-MarcosDiktatur" zu eigen.

\section{III.1 Risse im "Entwicklungsmodell "}

Die nach dem Aquino-Mord einsetzende Kapitalflucht führte dazu, daß Manila im Herbst 1983 den Reigen von Schuldenmoratorien eröffnen mußte. Es sah sich außerstande, die immensen Auslandschulden (26,5 Mrd. US-Dollar) zu bedienen und wurde genötigt, in intensive Umschuldungsverhandlungen mit den 483 Gläubigerbanken zu treten. Es dauerte über ein Jahr, bis Marcos und Premier- und Finanzminister Cesar Virata Mitte Oktober 1984 die Absichtserklärung unterschrieben, durch die sich Manila verpflichtete, allen Bedingungen zu genügen, um die Voraussetzungen für den Erhalt des 18. IWF-Standby-Kredits von 630 Mio. US- Dollar zu schaffen.

Die Zentralbank publizierte a m 14. November das Wirtschaftsmemorandum der Philippinischen Regierung, des IWF und des Internationalen Beratungskomitees, in dem sich Repräsentanten ausländischer Kreditinstitute zusammengeschlossen hatten. ${ }^{36} \mathrm{Ihm}$ zufolge sollte sich das reale Wirtschaftswachstum im Durchschnitt der Jahre 1984 bis 1990 auf $1,9 \%$ belaufen, wobei allerdings nach den niedrigen Werten der beiden ersten Jahre ab 1987 mit einer jährlichen Zuwachsrate von $4 \%$ gerechnet wird. Da gleichzeitig die Einwohnerzahl im Jahresdurchschnitt 1984-90 um 2,4 \% und die Erwerbsfähigenzahl um 3,5\% zunehmen, wird sich die wirtschaftliche Lage für das Gros der Bevölkerung in dieser Zeit noch verschlechtern und das Problem der Arbeitslosigkeit drückender werden (s. Tabelle auf S. 328).

Die Kreditvergabe, so die Vereinbarung, sollte in sieben Tranchen und vorbehaltlich einer positiven Bewertung seitens eines IWF-Teams erfolgen, das den mit dem Darlehen gekoppelten Diktat in bestimmten Intervallen (15. März, 15. August und 15. November 1985) zu überwachen hatte. Dermaßen einschneidend waren diesmal die Auflagen, daß man glauben konnte, über Nacht feiere das Lehnswesen als aktuelle Umgangsform zwischen Schuldner und Gläubiger eine verschämte Renaissance. Kurzfristig sollte Manila

- Preiskontrollen aufheben und eine "fortgesetzt gemäßigte Lohnpolitik" betreiben;

- Investitionsprogramme drastisch züsammenstreichen und das interne (direkte wie indirekte) Steueraufkommen um 13 Mrd. Pesos (ca. 650 Mio. US-Dollar) erhöhen;

- sämtliche Außenhandelsbeschränkungen beseitigen und die Interventionsmarge der Zentralbank beim Devisenhandel beschneiden und

36 Business Day (Quezon City) vom 15. November 1984; Philippine News and Features (Manila) vom 17. Dezember 1984, S. 4-7; Business Day vom 21. Januar 1985, S. 1/2 und vom 27. Februar 1985, S. 3. 


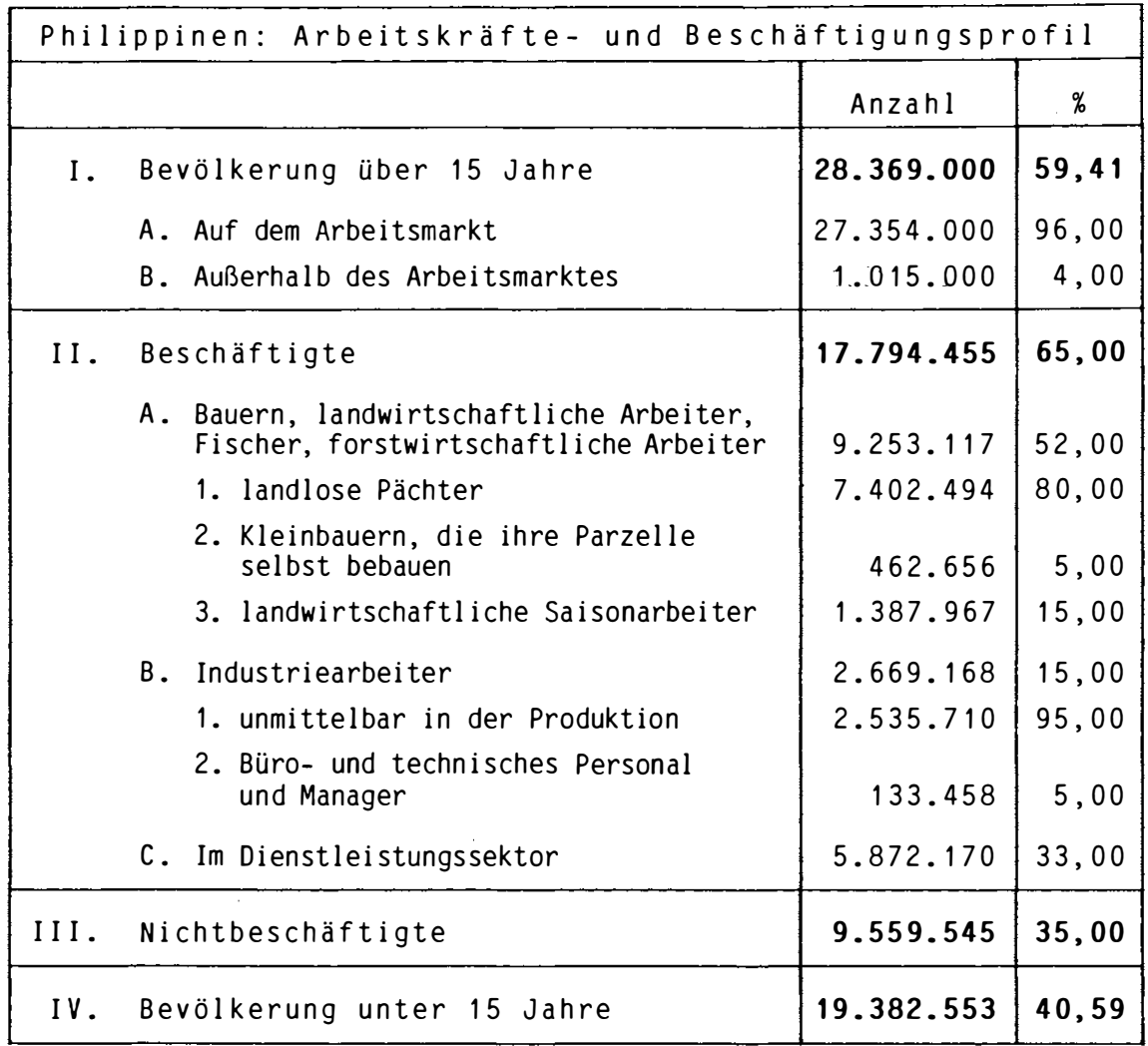

Quellen: Preliminary Report on Integrated Survey of Households (ISH), Labor Force: First Quarter to Fourth Quarter, 1979; NCSO (National Census and Statistics Office) Special Release No 335, NCSO/Philippines, 15 August 1980, und Kapit-Bisig, Conference Paper of the National Labor Assembly, Manila, Philippines, June 20, 1982.

- den Peso floaten sowie sämtliche Nahrungsmittelsubventionen (vorerst noch ausschließlich Reis) einstellen.

Die 630 Mio. US-Dollar verstanden sich als Teil eines 14 Mrd. US-Dollar betragenden Umschuldungspakets, das Manila in den kommenden Jahren zuzuschnüren hatte. Aus IWF-Sicht handelte es sich um ein recovery program. Die Malaise der Wirtschaft bildete das chronische Zahlungsbilanzdefizit, das Ende 1983 2,1 und Ende 1984 knapp 2 Mrd. US-Dollar ausmachte. Da eine Hauptkomponente der Zahlungsbilanz die Handelsbilanz darstellt, galt es nach Meinung der IWF-Experten, den Export weiter anzukurbeln, positive Handelsbilanzen herzustellen und letztlich die zur Schuldenbedienung erforderlichen Devisen zu erwirtschaften. 
Bei näherer Betrachtung des Ex- und Importprofils ergab sich freilich, daß die Einfuhren wesentlich (über $50 \%$ ) aus Kapitalgütern und Olprodukten bestanden. Die Ausfuhren indes waren hochgradig importlastig, d. h., daß über $70 \%$ des Gesamtexportwerts aus notwendig zu importierenden Gütern für die Textil-, Elektronik-, extraktive und Landwirtschaftsbranche bestritten werden mußten. Ungleich den unelastischen Einfuhrpreisen waren diejenigen $f$ ür Ausfuhren großen Schwankungen unterworfen. ZentralbankStatistiken wiesen aus, daß z. B. die Kokosnußöl-Exporte (die Inseln sind der Welt größter Kopraproduzent und -exporteur) zwar aufgrund weltweit gestiegener Nachfrage um $13 \%$ wuchsen, besagtes Produkt hingegen nur für durchschnittlich 513 US-Dollar pro Tonne statt des noch 1979 erzielten Tonnenpreises von 719 Dollar absetzbar war.

\section{III.2 Steigende absolute und relative Verarmung}

Mit mehr als $60 \%$ erreichte die Inflationsrate 1984 regionale Rekordhöhe. Das Bruttosozialprodukt verzeichnete einen Rückgang von $6 \%$, ein gleichsam in der Region konstatierbarer Negativrekord. 1984 mußten 1500 Firmen Bankrott anmelden und die Werkstore schließen. Uber 90000 Beschäftigte wurden auf die Straße geworfen, womit die Arbeitslosenquote - zumindest in Metro Manila - auf die $50 \%$-Marke zusteuerte. $\mathrm{Da}$ Manila sie weitaus niedriger ansetzte, resultierte aus seiner aberwitzigen Definition von Beschäftigung; danach galt bereits jeder als beschäftigt, der pro Quartal einen Tag arbeitete!

Veranschlagten Regierungsbehörden das zum Leben notwendige Tageseinkommen einer durchschnittlich 6köpfigen Familie auf 110 Pesos (5,5 US-Dollar), so betrug der gesetzlich vorgeschriebene Mindestlohn 59 Pesos, der jedoch in den wenigsten Fällen gezahlt wurde.

Untersuchungen verschiedener UN-Behörden, der in Manila beheimateten Asiatischen Entwicklungsbank (ADB) wie der University of the Philippines bezifferten die Zahl der unterhalb bzw. an der Armutsschwelle lebenden Menschen auf 70-80\%. Den durchschnittlichen Kalorienverbrauch gaben dieselben Organisationen mit täglich ca. 1700 pro Kopf an - weit unter dem als notwendig eingestuften Verbrauch von 2020 pro Tag/ Person und bedeutend niedriger als die von der UN-Welternährungsorganisation (FAO, Rom) angesetzte 2350-Mindestmarke. ${ }^{37}$

\section{III.3 Intensives Krisenmangement}

Nach dem Aquino-Mord setzte seitens Washington ein intensives Krisenmanagement ein, um die wirtschaftspolitische und Legitimationskrise des Marcos-Regimes einzudämmen.

37 Eine detaillierte Analyse des Gesundheitswesens findet sich in: R. Werning, Berstender Bambus - Befreiungskampf und Gesundheitswesen auf den Philippinen. Hrsg. von medico international. Frankfurt/M. 1986. 
Mitverantwortlich war hier nicht zuletzt die angeschlagene Reputation des Militärs. Generalstabschef Fabian C. Ver und weitere 25 Soldaten mußten im Herbst 1984 demissionieren, da sie der "Verwicklung" in den Aquino-Mord angeklagt waren. Vers Position wurde von Generalleutnant Fidel Ramos eingenommen, einem an der renommierten USMilitärakademie West Point ausgebildeten Eliteoffizier mit Kampferfahrungen in Korea und Vietnam. Von ihm wurde erwartet, daß er das durch Korruption und Inkompetenz gebeutelte Militär neuerlich als schlagkräftiges Instrument zur effektiven Aufruhrbekämpfung gestaltete. ${ }^{38}$ Gleichzeitig war beabsichtigt, partielle Dezentralisierungstendenzen einzuleiten - die counterinsurgency sollte eine zivile Komponente erhalten, indem politische Instanzen auf zumindest Provinzebene größeres Mitspracherecht eingeräumt wurde.

Seit Anfang 1984 bereisten hochrangige amerikanische Militärs, Politiker, Geheimdienstler und Bankiers den Archipel, ${ }^{39}$ um sich vor Ort ein Bild vom Ausmaß der Guerillaaktivitäten zu machen. Diesen Trend umzukehren, galt fortan die Stoßrichtung der Inter-Agency Taks Force on the Philippines. Diesem sich regelmäßig treffenden, behördenübergreifenden Gremium aus Repräsentanten des Pentagon und State Department, des Weißen Hauses und Schatzamtes sowie der CIA oblag die Formulierung einer vis-à-vis den Philippinen stromlinienförmig abgestimmten Politik. Unstimmigkeiten bzw. unterschiedliche Sichtweisen innerhalb der Task Force erschwerten zeitweilig den Entwurf einer einheitlichen Philippinen-Politik.

Verkürzt ließen sich diese Risse wie folgt charakterisieren: Das Schatzamt, der IWF und das Weiße Haus waren primär an der wirtschaftlichen Gesundung interessiert und konzentrierten sich erst in zweiter Linie auf die Uberwindung der politischen und Legitimationskrise. Donald Regan, zu der Zeit Finanzminister und gegenwärtig Stabschef im Weißen Haus, brachte diese Option auf einen griffigen Nenner:

"Ich vertrete nicht den Standpunkt, daß wir eine Nation nur deshalb vom Angelhaken (hook) springen lassen sollen, weil wir für deren Schwierigkeiten Sympathien hegen. ( . . . ) Als Gläubiger, so meine ich, sollten wir sie (die Philippinen; R. W.) dazu bringen, so viel wie möglich (an Auslandsschulden; R. W.) zurückzuzahlen, ohne deren Genick zu brechen. Es geht doch nicht an, in einer derartigen Situation Herz über Hirn triumphieren zu lassen. ${ }^{40}$

38 S. die Memoranden: Situation in the Philippines and Implications for U.S. Policy. Statement to the Committee on Foreign Relations, U.S. Senate, by James A. Kelly, Deputy Assistant Secretary of Defense (East Asia and Pacific Affairs), Washington, D.C. (18. 9.) 1984, 7 S.; das unter gleichem Titel verfaßte Statement to the Subcommittee on Asian and Pacific Affairs, Committee on Foreign Affairs, House of Representatives, by Richard L. Armitage, Assistant Secretary of Defense for International Security Affairs, Washington, D.C. (4. 10.) 1984, 10 S. und On the Insurgency Problem in the Philippines, by R. L. Armitage. Manila (12. 1.) 1985.

39 Dazu zählten: der Oberkommandierende der US-Streitkräfte im Pazifik, Admiral William Crowe; Washingtons Botschafter in Manila, Stephen Bosworth; der demokratische Kongreßabgeordnete Stephen Solarz; Paul Wolfowitz, im State Department verantwortlich für ostasiatische und pazifische Angelegenheiten; die Pentagonbeamten Kelly und Armitage, CIA-Direktor William Casey-sowie Senator Paul Laxalt.

40 Zit. nach: Walden Bello, Reagan's Philippine Policy: Preparing a U.S. Marcos-Dictatorship Without Marcos, in: Philippine Report (Berkeley, Calif.), November 1984, S. 4. 
Obgleich Präsident Reagan seinen nach dem Aquino-Mord geplanten Südostasien-Besuch abblies, leistete er sich während eines Fernsehduells mit seinem Herausforderer bei den letzten Präsidentschaftswahlen, Walter Mondale, einen bemerkenswerten Flop. Er würde nicht zulassen, so Reagan im Dezember 1983, "sie (die Marcos-Regierung; R. W.) den Wölfen (den Kommunisten; R. W.) vorzuwerfen und dann das Entstehen einer kommunistischen Macht im Pazifik zu dulden. "41 $^{4}$

Diese Argumentation wertete das State Department als unzulässig eindimensional, klammerte sie doch kategorisch die bürgerlich-gemäßigte Opposition in Gestalt der UNIDO (Vereinigten Nationalistischen Demokratischen Organisation) unter Vorsitz von Salvador H. Laurel und die von Kardinal Sin geführte Kirchenhierarchie gänzlich aus. Einen wie immer auch gearteten Aufwertungsversuch der Marcos-Regierung wertete das State Department angesichts der gesellschaftlichen Polarisierung als politisch kontraproduktiv. Stattdessen schenkte es der UNIDO höchste Aufmerksamkeit und bemühte sich aktiv um deren Hoffähigkeit. In diesem Sinne nahm es nicht wunder, daß Washingtons Botschafter in Manila, Stephen Bosworth, seit der Zeit mehrmals demonstrativ mit Politikern der UNIDO und ihr nahestehender Gruppierungen zusammentraf.

Das Pentagon bezog eine schillernde Position. Einerseits zog es seit Herbst 1983 erstmals die Verlagerung der im Lande installierten und größten außerhalb Nordamerikas befindlichen Militärbasen nach Mikronesien (Saipan. Tinian und Belau) in Betracht, ${ }^{42}$ plädierte aber nebst der ohnehin zwischen 1985 und 1989 gewährten Militär- und Wirtschaftshilfe für die Nutzungsrechte der Stützpunkte Subic Naval Base und Clark Air Field in Höhe von 900 Mio. US-Dollar für die Bereitstellung zusätzlicher Gelder im Rahmen von Oplan Katatagan. Daß es eine harte Tour verfocht, einem begrenzten und direkten US-Engagement bei der Counterinsurgency der philippinischen Streitkräfte nicht abgeneigt war, verwunderte kaum. Daß es aber eine materielle Stärkung durch eine persönliche Bevorzugung des auf Schlagkrafterhöhung bedachten Ramos zu Lasten des Marcos-Intimus Ver ergänzt sehen wollte und im ungebrochenen Krisenzustand eine über die politische Radikalisierung vermittelte potentielle Gefährdung der Militärbasen witterte, unterstrich seine Skepsis gegenüber einem rein militärisch motivierten Power Play.

Dennoch bestand innerhalb der Task Force ein Grundkonsens: Nebst dem militärstrategischen und für den Westen "ordnungspolitischen" Aspekt ist das Land ökonomisch von Bedeutung. Hier konzentriert sich das Interesse der USA auf den Schutz ihrer (auf ca. 4 Mrd. US-Dollar geschätzten) Direktinvestitionen, auf die Sicherung ihrer dominierenden Stellung im extraktiven, pharmazeutischen, Ol-, Transport- und Finanzsektor, auf den Erhalt der ausgedehnten Zitrusfruchtplantagen solcher Firmen wie Castle \& Cooke und United Brands (Mindanao) sowie auf die in den Exportproduktionszonen lukrative Billiglohnpolitik.

41 International Herald Tribune (Zürich/Paris) v. 26.-28. Oktober 1984.

42 Vgl. u. a.: William H. Sullivan, Living Without Marcos, in: Foreign Policy 53 (Winter 1983/84): 150-6, Washington, D.C.; ders., Relocating Bases in the Philippines, in: Washington Quarterly 7.2 (Frühjahr 1984) und The Philippines: Another Iran?, in: Newsweek (New York) v. 4. 11. 85, S. 26-37. 
Schließlich fällt dem Archipel im Rahmen des sich von Australien über Mikronesien und Südostasien nach Taiwan, Südkorea und Japan erstreckenden Pacific Rim (Pazifischen Beckens) die Funktion eines cordon sanitaire zu. Seit Beginn der 80er Jahre hat diese Region Europa den Rang als der USA größter Handelspartner abgelaufen. ${ }^{43}$ Japan verfolgt hier eigene, jedoch mit Washington abgestimmte Interessen - z. B. das Offenhalten der für es sensiblen (Ö)Nachschublinien wie die Straße von Malakka und das Südchinesische Meer. Im August 1985 kündigte Japans Ministerpräsident Yasuhiro Nakasone an, daß Tokio die lange Zeit als Hemmschwelle akzeptierte Begrenzung seiner Ausgaben für die Selbstverteidigungs-Streitkräfte auf weniger bzw. maximal ein Prozent des Bruttosozialprodukts bald zu überspringen gedenke.

Dieser von den Task Force-Parteien akzeptierte Konsens, gepaart mit dem emphatischen Bestreben, der in 62 der 73 Landesprovinzen verankerten Guerilla offensiver entgegenzutreten, dürfte für das Zustandekommen einer arbeitsfähigen Kompromißformel verantwortlich gewesen sein.

Ein delikates Amalgam aus ökonomischen, politischen und militärischen Elementen lieferte schließlich der von den Beteiligten Ende 1984 ausgearbeitete und dem Nationalen Sicherheitsrat weitergeleitete Philippinen-Plan, den Reagan im Januar 1985 als nunmehr gegenüber dem Archipel verbindliche National Security Directive (NSD) unterzeichnete. ${ }^{44}$

In ihr war ein Bündel von 16 "hohe Priorität genießenden Veränderungen" anvisiert, um die Gefahr zu bannen, daß "ein radikalisiertes Philippinen die gesamte Region destabilisiert." Von diesen Veränderungen wurde angenommen, daß "die gegenwärtige Regierung (sich) vielen von ihnen widersetzen wird." Dazu zählten z. B. eine weniger rigide Amtsführung des Präsidenten, d. h. Abschaffung seiner mit dem Preventive Detention Act (PDA) $^{45}$ geschaffenen legislativen Sondervollmachten sowie das Aufbrechen des Zucker- und Kopramonopols. Sibyllinisch hieß es in diesem Dokument: "Präsident Marcos ist Teil des Problems, aber auch Teil seiner Lösung."

Hinter einer solchen Sprachregelung verbarg sich ein Affront gegen den Herrscher im Malacañang-Palast. Strategisch, im Sinne einer geordneten Nachfolgeregelung erwünscht, war of fensichtlich eine Allianz des auf Reformen im Militär bedachten Ramos mit gemäßigten Politikern (aus dem UNIDO-Spektrum), wobei den aufgrund ihrer Kontakte zu internationalen Finanzkreisen reputierlichen Technokraten die Rolle eines Scharniers zukam. Deren Aufwertung erfolgte mit dem parallel orchestrierten Bemühen, Marcos auf snap elections - vorgezogene Präsidentschaftswahlen - zu drängen. CIA-Direktor William Casey hatte dies dem Präsidenten während seines Philippinen-

43 Eberhard Rhein, Die pazifische Herausforderung: Gefahren und Chancen für Europa, in: Europa Archiv, Zeitschrift für Internationale Politik, Folge 4/1984, Bonn.

44 National Security Study Directive: U.S. Policy Towards the Philippines, Washington, D.C. (Nov. 1984), 23 S. und Far Eastern Economic Review v. 21. 3. 85, S. $16 \mathrm{f}$.

45 Internationale Juristenkommission: Philippinen. Die Menschenrechte nach der Aufhebung des Kriegsrechts. epd-Dokumentation Nr. 22 und 23/85, Frankfurt/M. (Mai) 1985. 
Besuchs im Mai 1985 "nahegelegt".46 Spätestens im Oktober war erkennbar, daß Marcos sich diesem Ansinnen nicht länger mehr verschließen konnte. Reagans Sonderbeauftragter, Senator Paul Laxalt, war in jenem Monat nach Manila gereist, um diese Forderung mit einer Kritik an der Amtsführung des Präsidenten zu verknüpfen.

Als kurz darauf der Durenberger-Report ${ }^{47}$ ein vernichtendes Verdikt gegen die MarcosAdministration aussprach und in deren ungebrochener Herrschaft die Machtübernahme des NDF-Oppositionsbündnisses binnen drei Jahren für möglich hielt, ${ }^{48}$ blieb Marcos nurmehr die Flucht nach vorn. Gegen snap elections, so ließ er verlauten, habe er selbst-redend nichts einzuwenden.

Politisch war er unhaltbar geworden und wirtschaftlich galt der Archipel als unsicherster Kantonist in der Region, ein Urteil, dem sich die auf die Analyse von Kapitalanlagemöglichkeiten in Südostasien spezialisierte Economic and Political Risk Consultancy, Ltd. (Hongkong) anschloß.

Verzögerte Auszahlungen bereits zugesicherter Kredittranchen der IWF-WB-Gruppe und die "präventive" Stationierung amerikanischer Spezialeinheiten (SOF) in Clark und Subic unterstrichen den Anfang vom Ende der so lange ungetrübten bilateralen Beziehungen. ${ }^{49}$

\section{Die Präsidentschaftswahlen im Februar 1986}

Im Gegensatz zu den Parlamentswahlen vom Mai 1984 ging es jetzt nicht um eine "frische" Legitimation eines Regimes, dessen Image nach dem Aquino-Mord ramponiert worden war. Für die gemäßigte, bürgerliche Opposition war die Wahl als Testfall und für die Linke als soft option der Counterinsurgency im Sinne des Durenberger-Reports konzipiert, um den vom Parlament der Straße und der Guerilla ausgehenden Druck auf Wahlprozesse zu lenken.

Stephen Bosworth, Washingtons Botschafter in Manila, konferierte mehrmals mit Generalleutnant Fidel V. Ramos und dem oppositionellen Kandidatengespann Corazon C. Aquino und Salvador H. Laurel. Die auf Drängen der USA und Kardinal Sins in letzter Minute zustandegekommene Einigung zwischen Aquino und Laurel, auf einer gemeinsamen (UNIDO-)Plattform anzutreten, verhieß Gutes. In dieser zu Marcos höchst willkommenen personalen Alternative verbanden sich gewiefte Politik (Laurel) ${ }^{50}$

46 Newsweek v. 12. 8. 85, S. 16.

47 The Philippines: A Situation Report. Staff Report to the Senate Select Committee On Intelligence, United States Senate, Washington, D.C. (1. 11.) 1985, 18 S. - Vorsitzender dieses Komitees ist David Durenberger.

48 Ebda. und Düstere Prognosen für die Philippinen unter Marcos, in: Frankfurter Rundschau v. 4. 11. 85, S. 2.

49 RP (Republic of the Philippines) may call in US troops, in: Malaya (Quezon City) v. 1. 6. 85, S. 1; US policy veers towards military solution to Philippine insurgency, in: Business Day v. 2. 9. 85, S. 27 und Prof. Roland G. Simbulan, U.S. Intervention in the Philippines, (mimeo.), 1985, o.O., $4 \mathrm{~S}$.

50 Laurel entstammt einer einflußreichen Großgrundbesitzerfamilie aus der südlich von Manila gelegenen Provinz Batangas. Bis zu Beginn der 80er Jahre fühlte er sich Marcos eng verbunden. Erst seit dem Aquino-Mord ging er zu ihm auf Distanz und hegt seitdem eigene präsidiale Ambitionen. 
mit Integrität und Moral (Aquino), was angesichts des isolierten und verhaßten Regimes positiv zu Buche schlagen mußte. Diese Anti-Marcos-Konstellation bot zudem die Gewähr dafür, daß die amerikanischen Machtprärogativen tabu blieben.

Eine Aufwertung erfuhr auch die im Frühjahr 1985 erstmals ins öffentliche Bewußtsein gerückte Reformbewegung der Streitkräfte (RAM). Ihr Slogan, Loyalität gegenüber der Verfassung höher als die zu einer bestimmten Person/engruppe zu veranschlagen, fügte sich nahtlos in das Pentagon-Kalkül ein, gegen das noch von Ver befehligte Militär ein internes oppositionelles Gegengewicht zu stützen, das sich überdies auf eine effektive Guerillabekämpfung einrichtete. Ob amerikanische Militärs direkt am Aufbau der RAM beteiligt waren, ist bis dato nicht nachweisbar. Sicher indes ist, daß die RAM den in diversen amerikanischen Positionspapieren entfalteten Vorstellungen Rechnung trug. ${ }^{51}$

Aus alledem ergab sich eine Allianz drei gewichtiger Machtblöcke - UNIDO; RamosFraktion des Militärs und Kirchenhierarchie -, die gemeinsam den Sturz des Diktators besiegeln konnten. Die Technokraten des Regimes waren relativ mühelos zu neutralisieren bzw. von der Funktionsfähigkeit der neuen Allianz zu überzeugen. Schließlich zeichnen sie sich durch eine fehlende (partei)politische Basis und eine nur der Entwicklung verpflichteten Ideologie aus.

\section{Quo Vadis?}

Marcos' Sturz resultierte aus einer Kombination massiven Volkswiderstandes und der Abkehr wesentlicher Teile des Militärs, was den relativ friedlichen Ubergang zur Aquino-Regierung gestattete. Daß die Linke, vor allem die NDF, durch ihren Boykottaufruf und die Charakterisierung der Wahlen als "gigantischen Schwindel" die tiefsitzenden antidiktatorischen Sentiments des Volkes mißdeutete bzw. unterschätzte, hat in ihr einen Prozeß ebenso schmerzlicher wie heilsamer Kritik ausgelöst. Schmerzlich - weil die in ihr zusammengeschlossenen Organisationen maßgeblichen Anteil an der Erosion der Diktatur hatten, doch in den entscheidenden Februartagen außen vor blieben. Heilsam weil dieses Versäumnis eine intensiv geführte Diskussion entfachte, um Rigiditäten und Schematismen aufzubrechen. ${ }^{52}$

Aufgrund der o. g. Besonderheiten ihres Machtantritts ist die Aquino-Administration eine fragile Allianz aus personalen Restbeständen der Diktatur, ${ }^{53}$ der altneuen Militärspitze sowie aus konservativen und liberaldemokratischen Elementen der Anti-Marcos-

Die Ironie der Geschichte: Es war der politisch einflußreiche Laurel-Clan, der bis Mitte der 60er Jahre die Maschinerie der Nationalistischen Partei dominierte und Marcos in sein Camp herübergezogen hatte.

51 Vgl. die Anmerkungen 38, 44 und 47.

52 Vgl. z. B. Party conducts assessment, says boycott was wrong, in: Ang Bayan (Zentralorgan der CPP) 18. 3. (Mai 1986): 1-3, o.O.

53 Eine Vorstellung der Minister des Aquino-Kabinetts findet sich in: Südostasien aktuell Nr. 3/86, S. $200-1$. Hamburg (März) 1986. 
Front. ${ }^{54}$ Letztere genossen in den bewegten Februartagen besondere Aufmerksamkeit, als sie im Zuge freigesetzter Masseneuphorien die Freilassung der politischen Gefangenen - zumindest in Manila - erwirken konnten.

Ob und inwieweit es ihnen gelingt, die im Hinterland noch weitgehend intakten diktatorialen Strukturen (Bürokratie, Militär und Bandenwesen) zu schleifen, ist noch lange nicht entschieden. Frau Aquino und die Liberalen sind, sofern ihre politische Praxis über die Einhaltung der Menschenrechte hinaus auf soziale und ökonomische Strukturveränderungen in Stadt und Land abzielt, auf die Unterstützung der Linken angewiesen, wie denn auch diese im Interesse eines demokratischen Neubeginns nur zusammen mit jenen Kräften ein wirksames Gegengewicht zu den Architekten eines Thermidors zu schaffen vermöchten.

Gelänge das, so hätte der in den Februartagen des Jahres 1986 eilfertig geprägte Begriff People Power tatsächlich seine Berechtigung und eine Signalwirkung auf Diktaturen ähnlicher Provenienz. 


\section{Japan: The Conservative Revolution}

\section{By Ludger Kühnhardt}

The roots of modern Japanese society have been established during the 19th century. The Meiji-Era has been considered as one of the most interesting historical transformations in world history. The Japanese started to modernize their society along the lines of Western methods and techniques while rejecting the very foundations of Western civilization, Christian religion and the individualistic philosophies of the Age of Enlightenment in particular. After 250 years of Shogunal rule, the Meji-Restoration provided for the shift in political power in favour of the imperial régime. 1868 became the turning point of Japanese history. The remarkable historical development which followed transformed Japan into one of the leading world powers. It would, however, be misleading to describe Japan as a Westernized country. The opposite is true: notwithstanding the Meji-Era and the fundamental changes in the social, political and cultural structure of the country and its society after the Second World War, Japan remains basically an Oriental society, almost anti-Western with respect to the foundations of its intellectual fabric and notions of the individual. The article describes the historical and intellectual development of Japan with particular emphasis on the Meiji period which ended with the promulgation of the Meiji constitution in 1889: it provided Japan as the first Asian country with a modern constitutional system.

\section{The Philippines: An Anatomy of the Rise and Fall of a Dictatorship}

\section{By Rainer Werning}

This paper describes the reign of the former Philippine dictator Ferdinand E. Marcos, the political aims and interest groups guiding his régime, and the emergence of the succeeding administration led by Corazon Aquino.

Intricate internal coalitions as well as foreign involvement, especially of the United States of America, continue to subtend the new Aquino government while the radical opposition to the Marcos oligarchy has been largely bypassed by the post-Marcos political dispensation. 